\title{
Minimizing Musculoskeletal Disorders THROUGH FUZZIFIED NEURAL NETWORK APPROACH
}

\author{
Aman Sachdeva ${ }^{1}$, B.D.Gupta ${ }^{1}$, Sneh Anand ${ }^{2}$ \\ ${ }^{1}$ Dept of Mechanical Engineering, Anand Engineering College, Agra (India) \\ amansachdeva@hotmail.com, Bdgupta_aec@hotmail.com \\ ${ }^{2}$ Centre for Bio-Medical Engineering, Indian Institute of Technology, Delhi (India) \\ Snehanand_ittdehotmail.com
}

\begin{abstract}
In casting units, workers work under tough conditions to perform the desired task. These tough conditions normally give rise to various musculoskeletal disorders within the workers. These disorders emerge within the workers body due to repetitive lifting, differential lifting height, ambient conditions etc. For the minimization of musculoskeletal disorders it is quite difficult to model with mathematical difference or differential equations. In this paper the minimization of musculoskeletal disorders problem has been formulated using artificial neural networks model. But the existing neural network model has various drawbacks like large training time, huge data requirement to train for a non linear complex musculoskeletal disorders problem, the relatively large number of hidden nodes required etc. Hence, an attempt has been made to develop a non linear model using fuzzified neuron sub-models to overcome the above mentioned problems. These models would have the capability of representing solutions for minimizing musculoskeletal disorders needed for workers working in the casting units.
\end{abstract}

\section{KEYWORDS}

ANNs Model, Musculoskeletal disorders, Neural Networks

\section{INTRODUCTION}

The ability to maintain balance between health and production targets is increasingly important within workers of different industry. Workers tend to exert their bodies to various types of musculoskeletal disorders unknowingly and tend to lose both on the financial end as well on the health end. Generally these types of disorders tend to decrease the efficiency of the workers over a length of time especially for those who are highly exposed to repetitive tasks. The ligaments of the workers tend to depreciate over a period of time such that they lead to critical permanent disabilities [1-5]. The ligaments of the shoulder muscle as well as of the low back muscles are the major effected muscles among casting unit workers. Musculoskeletal disorders are the condition where a part of musculoskeletal system gets injured over time due to repetitive task. Musculoskeletal disorders are the disorders where the human body gets traumatized in either a minor or major way over a period of time. Most common examples of these type of disorders are: vibrating white finger, scoliosis etc. Musculoskeletal disorders are caused in most of the industries where the workers work day and night for their bread and butter without having any sufficient knowledge how to pursue their work so as to have the right balance between health and money earned. In this chapter the main focus will be to observe the impact of these musculoskeletal disorders on automobile industry in detail. Survey conducted in thirty odd automobile industry both of small scale and medium scale type, it is clear that musculoskeletal disorders had a wide existence within the poor unknown workers working day DOI : 10.5121/ijaia.2011.2307 
International Journal of Artificial Intelligence \& Applications (IJAIA), Vol.2, No.3, July 2011

and night. Consequences of these disorders was huge as it was observed in the survey that in many cases the poor workers had to quit from their job permanently as their body had no more tolerance power to work anymore under the given circumstances. Normally it is observed that workers tend to exert their body to such undesirable levels that it became difficult for themselves even to bear the pain which occurred after some time.

Main Causes of MSD's that were observed in Casting Industry are:-

- Maintaining an unnatural or unhealthy posture while working.

- Lower back support is inadequate for the user.

- Continuous sitting in the same position for an extended period of time.

- Set up of the workstation is ergonomically poor.

- Adverse work environment.

- Continuous lifting of heavy automobile components from ground height.

- Awkward posture maintained by workers during lifting

These Musculoskeletal disorders represent a wide range of disorders, which can differ in severity from mild periodic symptoms to severe chronic and debilitating conditions [9-15]. The challenges in designing the workstation for repetitive lifting task workers can be broadly categorized as follows:

a. Optimizing differential lifting heights

b. Minimizing musculoskeletal disorders

c. Increasing the efficiency of workers

This paper concentrates on minimizing musculoskeletal disorders for workers of casting unit of automobile industry. Repetitive, forceful or prolonged exertions of the hands; frequent or heavy lifting, pushing, pulling, or carrying of heavy objects; prolonged awkward postures; and vibration contribute to Work based musculoskeletal disorders (WMSDs) [16-20].

The artificial neural networks and fuzzy set theoretic approach are very often used for complex problems, which are highly non-linear in nature like musculoskeletal disorder forecasting problem. The integration of these approaches can give improved results. Both the modelling techniques have their own advantages and disadvantage as follows:

1. Fuzzy models possess large power in representing linguistic and structured knowledge by fuzzy sets and performing fuzzy reasoning by fuzzy logic in qualitative manner and usually rely on the domain experts to provide the required knowledge for a specific problem.

2. The compensatory operators in the fuzzy models as connectives are found quite suitable and give results very close to the actual result.

3. On the other hand, neural network models are particularly good for non-linear mappings and for providing parallel processing facility to simulate complex system. The neural network models are developed via training.

4. Furthermore, while the behavior of fuzzy models can be understood easily due to their logical structure and step by step inference procedures, a neural network models acts normally as a black box, without providing explanation facility [6-8]. 
International Journal of Artificial Intelligence \& Applications (IJAIA), Vol.2, No.3, July 2011

From these investigations, it is quite natural to consider the possibilities of integrating the two paradigms, in order to utilize the desired strength of both types of models. There are various possibilities for combining fuzzy systems and neural networks such as:

1. Treating fuzzy model and neural network model as two different compositional elements. The overall task is divided into two, some of which are completed by neural networks and some others by fuzzy models.

2. Fuzzy systems may be employed as a supervisor at a higher level in the hierarchy and supervise lower level neural network models.

3. The other possibility of the combination of these systems is that both systems work together competitively or co-operatively

- By neuralizing fuzzy systems i.e. the introduction of neural network concepts in fuzzy systems. Technically, it may be realized by mapping out fuzzy systems into neural network, either functionally or structurally.

- Or fuzzifying neural networks implying introducing fuzzy concepts in neural networks. As is well recognized, fuzzy systems are logical based with fuzzy set representation and flexible fuzzy logic operations. Thus the resulting fuzzy neural system may include minimum, maximum or compensatory operators, apart from usual sum and product operators found in neural computing. For example, product - sum unit may be replaced by a min-max unit.

In this paper, an attempt has been made to develop a fuzzified neural network for musculoskeletal disorders forecasting problems. Conventional network model has been modified using fuzzy compensatory operators as aggregation operators to overcome the following obstacles of these models:

i. Number of neurons required in hidden layer is large for complex function approximation.

ii. Number of hidden layers required for complicated functions may be three or more.

iii. Above mentioned bottlenecks are not only computationally expensive in terms of convergence and large number of neurons in each layer, but also in determining fault tolerant capabilities of the neural network.Several iterations are required before the artificial neural network gets trained to give accurate results.

iv. Size of neural networks decides total number of unknowns to be determined and hence minimum number of training input - output pairs required for development of the model. In the case of complex functions, a huge set of training data is necessary due to the requirement of large number of neurons and hidden layers.

$\mathrm{v}$. The training time of the neural network depends on the input and output mappings like $\square \mathrm{x}-\mathrm{y}$, $\mathrm{x}-\square \mathrm{y}, \square \mathrm{x}-\square \mathrm{y}$ and so on. The effect of mappings and different normalization ranges has been studied for different electrical machine models and load forecasting problems adopting neural network [21-28]. The proposed fuzzified neuron models overcome these drawbacks and incorporate the following features:

i. They accommodate variations in mappings and hence drastically reduce the total number of neurons in the neural network.

ii. These neurons should also exhibit characteristics of existing neurons so that the models are general enough to accommodate properties of simple neurons to higher order neurons. 
iii. Total number of hidden layers required must also be reduced. This would result in a neural network model, which is computationally efficient.

iv. The new models should not require huge data for training, i.e. there should be reduction in free parameters associated in the neurons [29-31].

\section{EXISTING NEURON MODELS}

The general structure of the existing simple neuron is an aggregation function and its transformation through a filter. It has been shown in literature that the neural networks can be universal function approximators for given input-output data. However, the existing simple neuron structure has summation as aggregation function with sigmoidal, radial basis, tangent hyperbolic or linear limiters as thresholding functions as shown in Figure 1.
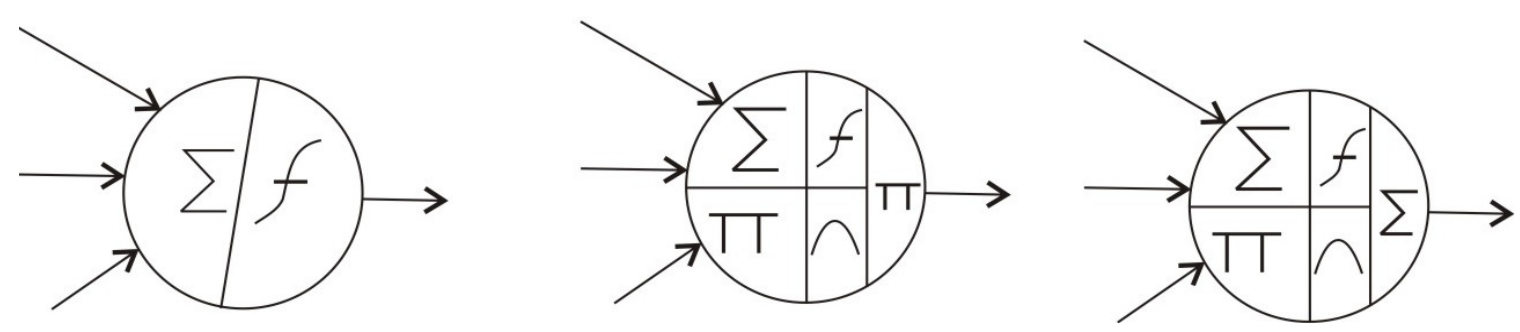

Figure 1 - Existing Neuron Model, Fuzzified Neuron Model-0 and Fuzzified Neuron Model-1

\section{DEVELOPMENT OF FUZZIFIED NEURON MODELS}

The aggregation operators used in the neurons are generally crisp. However, they overlook the fact that most of the processing in neural networks is done with incomplete information at hand. Thus a fuzzified neuron model approach has been adopted which uses the fuzzy compensatory operators, which are partly sum and partly product to take into account the vagueness involved, as described by Muzomoto in his paper. Existing models use the sigmoidal thresholding function and ordinary summation or product as aggregation functions. These fail to cope with the non-linearities involved in real life problems. To deal with the above, the proposed models have both sigmoidal and gaussian functions with weight sharing. The fuzzified neuron models have flexibility at both the aggregation and threshold function level to cope with the non-linearity involved in the type of applications dealt with. The neuron has both $\Sigma$ and $\pi$ aggregation functions. The aggregation function has been used with the Sigmoidal characteristic function while the aggregation function has been used with the Gaussian function as a characteristic function. The final output of the neuron is a function of the two outputs $\mathrm{O}_{\Sigma}$ and $\mathrm{O}_{\pi}$ with the weights $\mathrm{W}_{\Sigma}$ and $\mathrm{W}_{\pi}$, respectively. 
FORECASTED MSD USING MODEL BKP \& 1

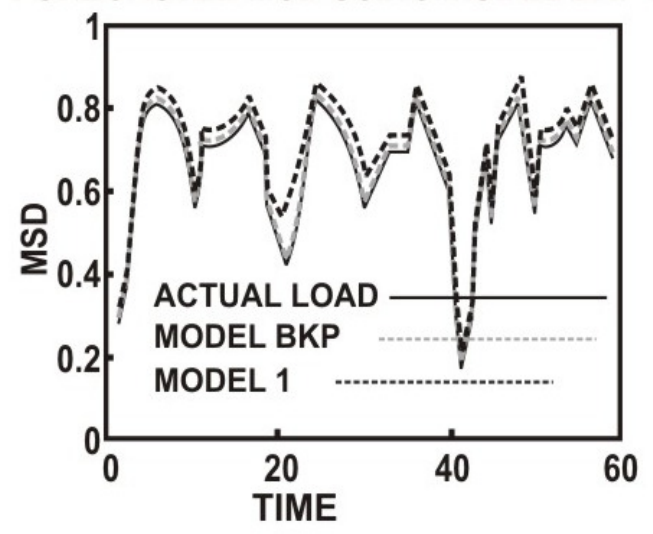

MODEL 4 \& 5

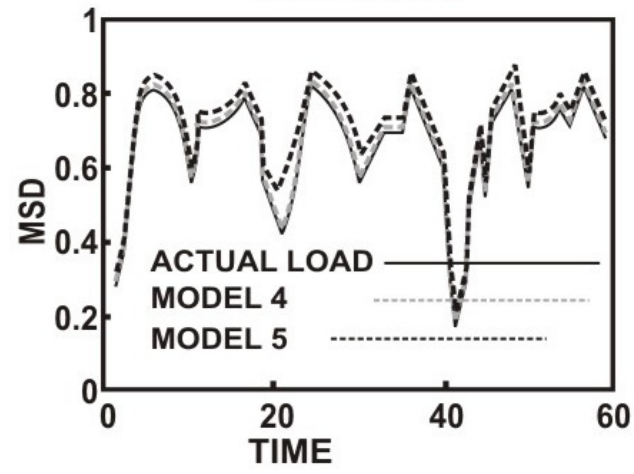

MODEL 2 \& 3

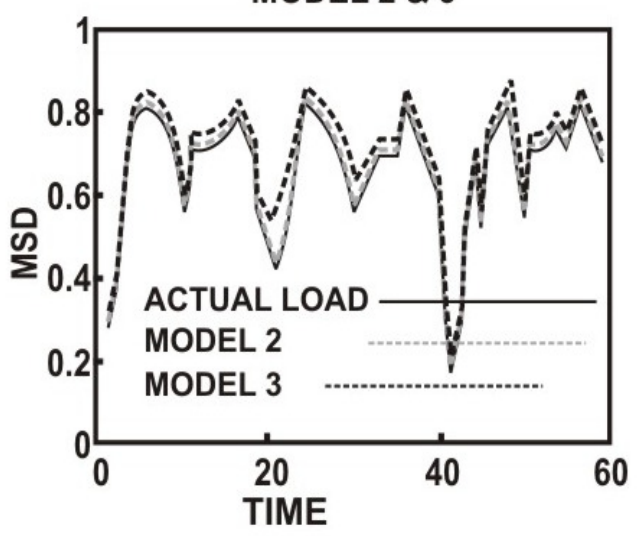

MODEL 6

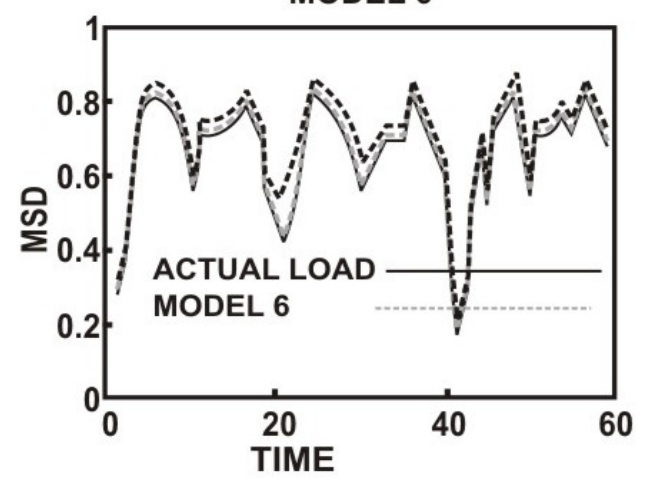

Figure 2 - Simulation results of Musculoskeletal Disorder Forecasting using existing simple neural network model and generalized neuron models 0-6

\subsection{Fuzzified neuron model-0}

In this model both $\Sigma$ as well as $\pi$ have been taken as the aggregation functions and the output of these aggregation functions have been passed through the Sigmoidal and Gaussian functions respectively. Finally, the outputs are summed up to get the neuron output. The output of the neuron can be mathematically written as:

$\mathrm{O}_{\mathrm{pk}}=\mathrm{O}_{\pi} * \mathrm{~W}_{\pi} * \mathrm{O}_{\Sigma} * \mathrm{~W}_{\Sigma}$

\subsection{Fuzzified neuron model-1}

This model is similar to the above developed generalized neuron model- 0 . The only difference is that in this model the weight associated with the output of the product aggregation function when passed through the Gaussian function is $\left(1-\mathrm{W}_{\Sigma}\right)$ as shown in Figure 2 . Hence the output of the neuron becomes:

$\mathrm{O}_{\mathrm{pk}}=\mathrm{O}_{\pi} *\left(1-\mathrm{W}_{\Sigma}\right) * \mathrm{O}_{\Sigma} * \mathrm{~W}_{\Sigma}$

The above mentioned neuron model is known as summation type compensatory neuron model, since the output of the Sigmoidal and Gaussian functions have been summed up. 


\section{LEARNING ALGORITHM OF GENERALIZED NEURONS}

\subsection{Model-1}

The following steps are involved in the training of Generalized Neural Network:

Step 1 - The output of $\Sigma$ - part of generalized neuron is:

$\mathrm{O}_{\Sigma}=\mathrm{f} 1\left(\Sigma \mathrm{W}_{\Sigma \mathrm{i}} \mathrm{Xi}+\mathrm{Xo}_{\Sigma}\right)$

Step 2 - The output of $\pi$ - part of generalized neuron is:

$\mathrm{O}_{\pi}=\mathrm{f} 2\left(\pi \mathrm{W}_{\pi \mathrm{i}} \mathrm{Xi}+\mathrm{Xo}_{\pi}\right)$

Step 3 - The output of Generalized Neuron can be written as:

$\mathrm{O}_{\mathrm{pk}}=\mathrm{O}_{\pi} *\left(1-\mathrm{W}_{\Sigma}\right) * \mathrm{O}_{\Sigma} * \mathrm{~W}_{\Sigma}$

Step 4 - after calculating the output of Generalized Neuron in the forward pass of feedforward backpropagation neural networks, it is compared with the desired output to find the error and then it is minimised to train the Generalized Neural Network (GNN) model. Hence in this step the output of the GNN with a single flexible generalized neuron model is to be compared with the desired output to get error for the ith set of input.

Error $\mathrm{E}_{\mathrm{i}}=\left(\mathrm{Y}_{\mathrm{i}}-\mathrm{O}_{\mathrm{i}}\right)$

Then it is necessary to calculate the sum squared error for convergence while training.

$\mathrm{E}_{\mathrm{p}}=0.5 \Sigma \mathrm{E}_{\mathrm{i}}^{2}$

A multiplication factor of 0.5 has been taken for simplifying the calculations.

Step 5 - Reversed pass for modifying the connection strength.

- Weight associated with the $\Sigma$ and $\Sigma$-parts of the Generalized Neuron

$\mathrm{W}_{\Sigma}(\mathrm{k})=\mathrm{W}_{\Sigma}(\mathrm{k}-1)+\Delta \mathrm{W}_{\Sigma}$

where $\Delta \mathrm{W}_{\Sigma}=\eta \delta_{\Sigma}\left(\mathrm{O}_{\Sigma}-\mathrm{O} \pi\right) \mathrm{Xj}+\alpha \mathrm{W}_{\Sigma}(\mathrm{k}-1)$

and $\delta_{\Sigma}=\Sigma(\mathrm{Yi}-\mathrm{Oi})$

- Weight associated with the inputs of the $\Sigma$-parts of the Generalized Neuron:

$\mathrm{W}_{\Sigma \mathrm{i}}(\mathrm{k})=\mathrm{W}_{\Sigma \mathrm{i}}(\mathrm{k}-1)+\Delta \mathrm{W}_{\Sigma \mathrm{i}}$

where $\Delta \mathrm{W}_{\Sigma \mathrm{i}}=\eta \delta \Sigma \mathrm{j} \mathrm{Xj}+\alpha \mathrm{W}_{\Sigma \mathrm{i}}(\mathrm{k}-1)$ and

$\delta_{\Sigma \mathrm{j}}=\Sigma \delta_{\mathrm{k}} \mathrm{W}_{\Sigma} \mathrm{O}_{\Sigma}\left(1-\mathrm{O}_{\Sigma}\right)$

- Weight associated with the input of the $\pi$-parts of the Generalized Neuron

$\mathrm{W} \pi \mathrm{i}(\mathrm{k})=\mathrm{W} \pi \mathrm{i}(\mathrm{k}-1)+\Delta \mathrm{W}_{\pi \mathrm{i}}$

where $\Delta \mathrm{W}_{\pi \mathrm{i}}=\eta \delta \pi \mathrm{j} \mathrm{Xj}+\alpha \mathrm{W} \pi \mathrm{i}(\mathrm{k}-1)$ and

$\delta \pi \mathrm{j}=\Sigma \delta \mathrm{k}\left(1-\mathrm{W}_{\Sigma}\right) \mathrm{O} \pi(-2 *(\pi \mathrm{WiXi}+\mathrm{Xo} \pi))$

\subsection{Fuzzified neuron model-2}

The neuron model-2 is not a summation neuron model but a product type compensatory neuron model. The output of the Sigmoidal and Gaussian functions have been multiplied after being exponentiated to the powers $\left(1-\mathrm{W}_{\Sigma}\right)$ and $\mathrm{W}_{\Sigma}$. The neuron structure and its detailed diagram 
have been shown in Figure 3. Here the output of this product type compensatory neuron is in the form of product as given below: $\mathrm{O}_{\mathrm{pk}}=\mathrm{O}_{\pi}^{(1-\mathrm{W} \Sigma)} * \mathrm{O}_{\Sigma}{ }^{\mathrm{W}}{ }_{\Sigma}$

RANGE 0.1 TO 0.9

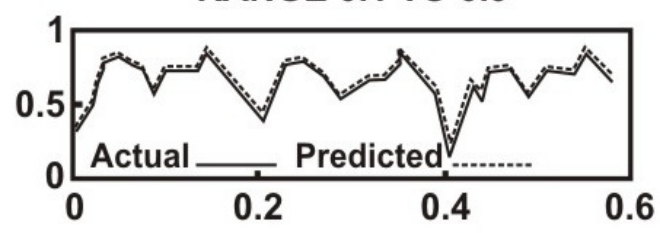

RANGE 0.1 TO 0.9

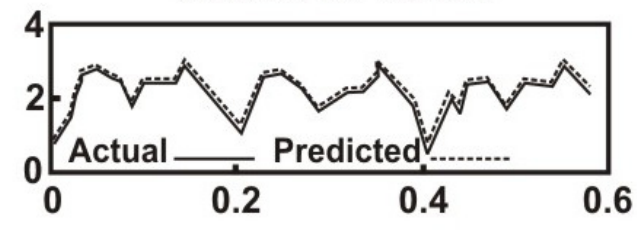

RANGE -0.9 TO 0.9

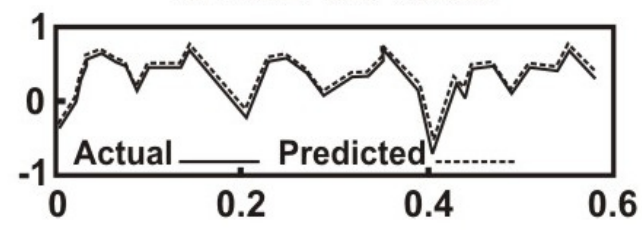

RANGE 0.1 TO 0.8

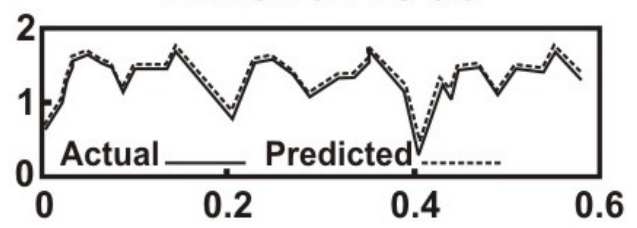

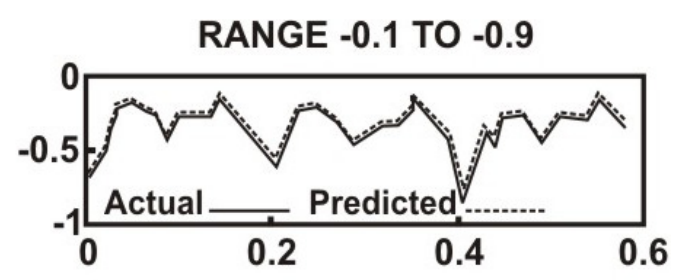
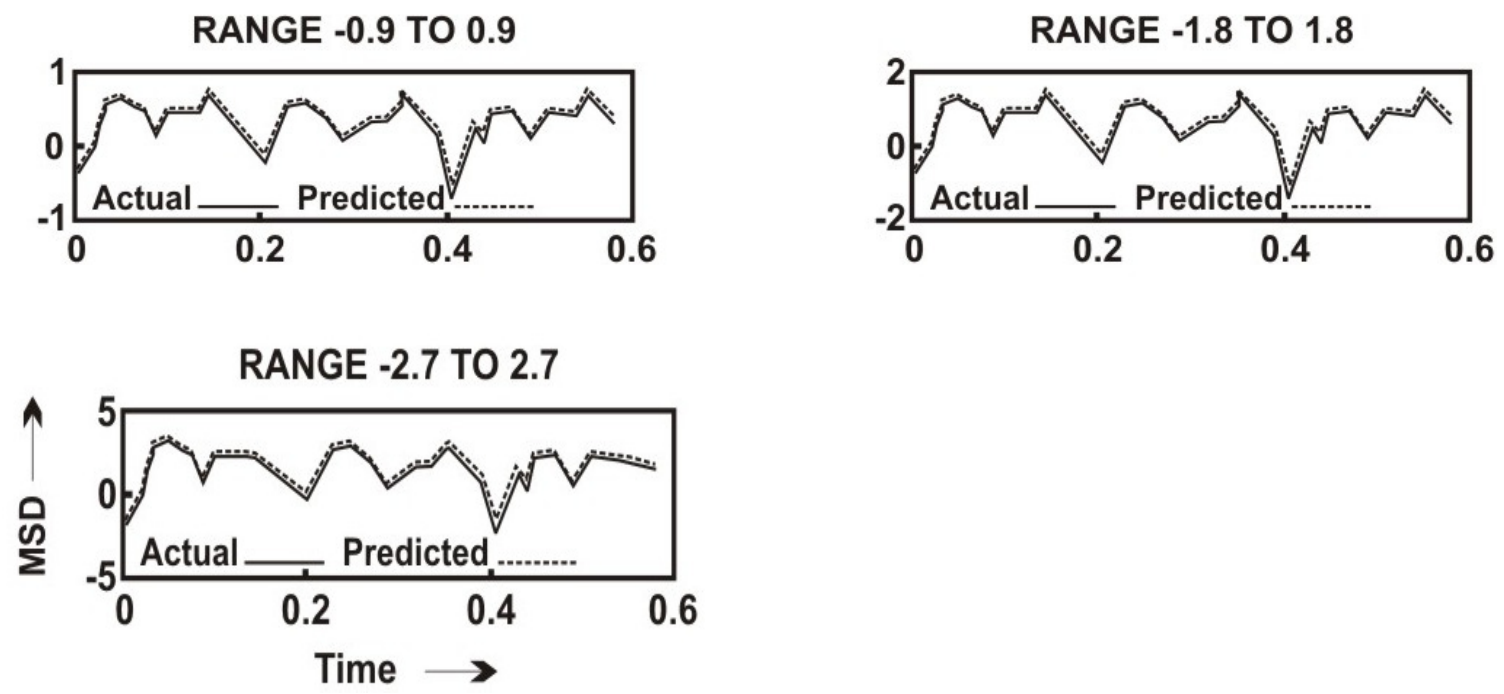

Figure 3 - Effect of Normalizations on the results of musculoskeletal disorder forecasting model using Generalized Neuron Model - 1

\subsection{Fuzzified neuron model-3}

This neuron model has a complicated aggregation function which is neither a summation function nor a product function alone but a summation type compensatory aggregation function. The output of this neuron model is:

$\mathrm{O}_{\mathrm{pk}}=\left(\mathrm{O}_{\Sigma}-\mathrm{O}_{\pi}-\mathrm{O}_{\Sigma} * \mathrm{O}_{\pi}\right)\left(1-\mathrm{O}_{\Sigma}\right) * \mathrm{O}_{\Sigma} \mathrm{W}_{\Sigma}$

\subsection{Fuzzified neuron model-4}

This model is similar to the above neuron model-3 but the output of the compensatory neuron is in the product form as given below:

$\mathrm{O}_{\mathrm{pk}}=\left(\mathrm{O}_{\Sigma}-\mathrm{O}_{\pi}-\mathrm{O}_{\Sigma} * \mathrm{O}_{\pi}\right)^{(1-\mathrm{W} \Sigma) / 2} * \mathrm{O}_{\Sigma} \mathrm{W}_{\Sigma}$ 


\subsection{Fuzzified neuron model-5}

This is also a summation type compensatory neuron model, however, it uses the arithmetic and geometric means of the output of sigma and the product aggregation functions.

The neuron output is as follows:

$\mathrm{O}_{\mathrm{pk}}=\left(\mathrm{O}_{\Sigma}-\mathrm{O}_{\pi}\right)\left(1-\mathrm{W}_{\Sigma}\right) / 2+\sqrt{ }\left(\mathrm{O}_{\Sigma} * \mathrm{O}_{\pi}\right) \mathrm{W}_{\Sigma}$

\subsection{Fuzzified neuron model-6}

This model is similar to the above developed generalized neuron model-5 but the output is in the product form as follows:

$\mathrm{O}_{\mathrm{pk}}=\left(\mathrm{O}_{\Sigma}-\mathrm{O}_{\pi}\right){ }_{\Sigma}^{\mathrm{w}} / 2 *\left(\mathrm{O}_{\Sigma} * \mathrm{O}_{\pi}\right)^{(1-\mathrm{W} \Sigma) / 2}$

\section{MUSCULOSKELETAL DISORDERS FORECASTING USING FUZZIFIED NEURON MODELS}

Experimentation was done on the SUBJECTS between 36 to 55 years and their EEG was recorded. SUBJECTS were asked to lift a $29 \mathrm{~kg}$ block from different height and put the block on the fixed platform which is at a height of 40 inches from the ground. The Lifting of the block was made synonymous with the actual task performed by the workers during casting of automobile products. Based on the lifting sessions their EEG was recorded and the value of the musculoskeletal disorder was taken based on the graphs variation from the mean. The values of the four inputs were normalized and were taken as the input data. EEG of the subject is shown in Fig 4. The results of the experiments were normalized as shown in Table 1 i.e.

Input 1 - Ambient Conditions

Input 2 - Frequency Of Lift

Input 3 - Angle of Twist

Input 4 - Lifting Height

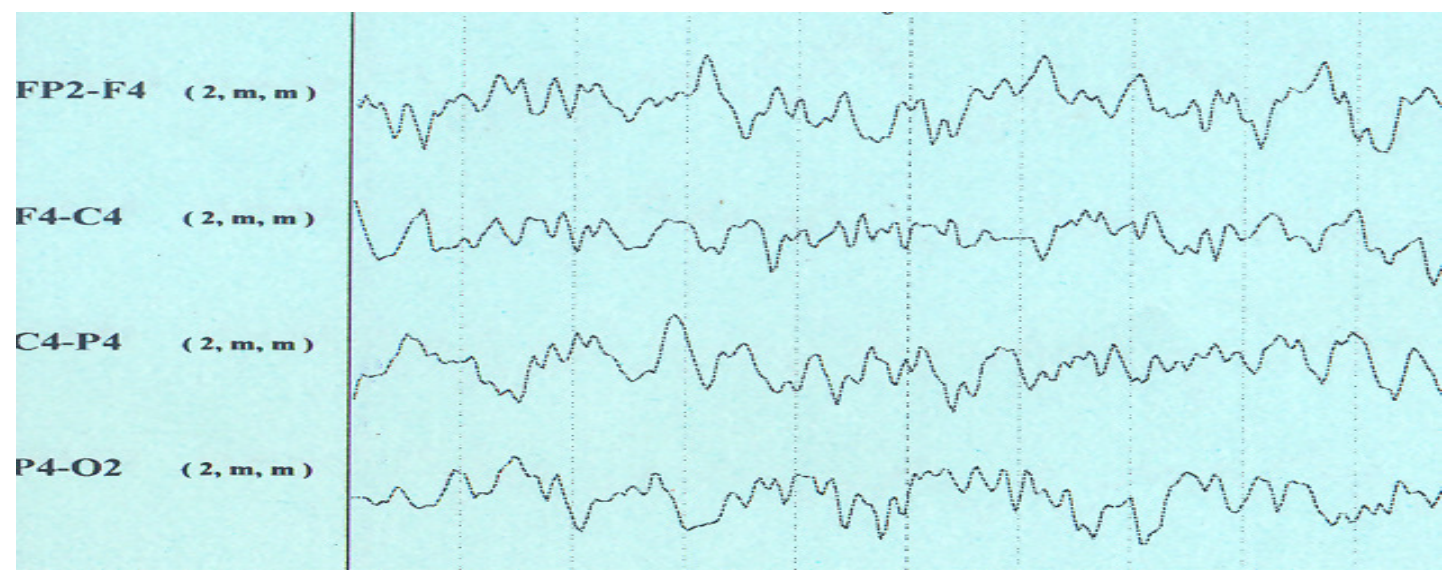

Fig 4. Electroencephalography of the SUBJECT

The parameters for generalised neural network models and existing neural networks are as follows: 
Learning rate -0.1

Momentum - 0.6

Gain scale factor -1.0

Tolerance -0.01

\section{Table 1 Training Data}

$\begin{array}{ccccc}\text { Input } 1 & \text { Input } 2 & \text { Input } 3 & \text { Input } 4 & \text { MSD } \\ 0.34 & 0.31 & 0.27 & 0.27 & 0.39 \\ 0.77 & 0.38 & 0.62 & 0.77 & 0.8 \\ 0.61 & 0.78 & 0.8 & 0.81 & 0.8 \\ 0.81 & 0.74 & 0.6 & 0.7 & 0.7 \\ 0.6 & 0.7 & 0.7 & 0.72 & 0.73 \\ 0.72 & 0.73 & 0.77 & 0.83 & 0.8 \\ 0.84 & 0.81 & 0.79 & 0.64 & 0.55 \\ 0.37 & 0.36 & 0.32 & 0.38 & 0.5 \\ 0.38 & 0.5 & 0.7 & 0.77 & 0.8 \\ 0.7 & 0.78 & 0.8 & 0.8 & 0.75 \\ 0.8 & 0.75 & 0.67 & 0.55 & 0.55 \\ 0.67 & 0.55 & 0.55 & 0.6 & 0.65 \\ 0.5 & 0.6 & 0.65 & 0.66 & 0.67 \\ 0.66 & 0.67 & 0.8 & 0.83 & 0.57 \\ 0.8 & 0.84 & 0.75 & 0.66 & 0.6 \\ 0.5 & 0.42 & 0.58 & 0.71 & 7000 \\ 0.64 & 0.53 & 0.63 & 0.67 & 0.73 \\ 0.63 & 0.67 & 0.73 & 0.7 & 0.7 \\ 0.7 & 0.7 & 0.8 & 0.85 & 0.8 \\ 0.8 & 0.86 & 0.8 & 0.73 & 0.65\end{array}$

The above mentioned generalized neural network models with only one neuron have been trained for the data given in Table 1 with the goodness of fit represented by tolerable sum squared error equal to 0.001 . The performance of various models in terms of training time (epochs) required is given in Table 2

Table 2 Comparison of training time for different neuron models for

Musculoskeletal disorder forecasting problem

(Tolerable Sum Squared Error = 0.0001)

$\begin{array}{ll}\text { Model } & \text { Epochs } \\ \text { Existing } & 850 \\ \text { Model 0 } & 760 \\ \text { Model 1 } & 760 \\ \text { Model 2 } & 336 \\ \text { Model 3 } & 250 \\ \text { Model 4 } & 370 \\ \text { Model 5 } & 650 \\ \text { Model 6 } & 200\end{array}$


The generalized neural network models have been tested for the data presented in Table 3 . The results obtained for musculoskeletal disorder forecasting problem from the generalized neural network using fuzzified neuron model $0-6$ and the existing neural network have been compared with the actual results and it is found that the generalized neural network is more efficient and accurate. The RMS, minimum and maximum errors for forecasting using different neuron models with X-Y mapping and 0.1 to 0.9 as the normalization range are tabulated in Table 4 . From this table, it is very clear that the neuron model-6 requires the least training epochs with model-1 gives the minimum RMS predictive error.

Table 3 Testing Data

\begin{tabular}{|c|c|c|c|c|c|c|}
\hline $\begin{array}{l}\text { Input } 1 \\
\text { ANN }\end{array}$ & Input 2 & Input 3 & Input 4 & Output & Results of GNN & Existing \\
\hline 0.34 & 0.31 & 0.27 & 0.27 & 0.38 & 0.36 & 0.38 \\
\hline 0.28 & 0.27 & 0.38 & 0.61 & 0.77 & 0.74 & 0.79 \\
\hline 0.27 & 0.38 & 0.61 & 0.77 & 0.79 & 0.80 & 0.79 \\
\hline 0.38 & 0.61 & 0.77 & 0.79 & 0.81 & 0.77 & 0.82 \\
\hline 0.61 & 0.77 & 0.79 & 0.81 & 0.81 & 0.77 & 0.78 \\
\hline 0.77 & 0.79 & 0.81 & 0.81 & 0.74 & 0.76 & 0.74 \\
\hline 0.81 & 0.74 & 0.60 & 0.69 & 0.70 & 0.70 & 0.73 \\
\hline 0.74 & 0.60 & 0.69 & 0.70 & 0.71 & 0.69 & 0.73 \\
\hline 0.60 & 0.69 & 0.70 & 0.71 & 0.72 & 0.72 & 0.76 \\
\hline 0.7 & 0.7 & 0.71 & 0.72 & 0.77 & 0.71 & 0.74 \\
\hline 0.72 & 0.72 & 0.77 & 0.83 & 0.81 & 0.78 & 0.79 \\
\hline 0.73 & 0.77 & 0.83 & 0.81 & 0.78 & 0.75 & 0.74 \\
\hline 0.84 & 0.81 & 0.78 & 0.64 & 0.55 & 0.60 & 0.63 \\
\hline 0.34 & 0.37 & 0.36 & 0.32 & 0.37 & 0.39 & 0.50 \\
\hline 0.37 & 0.36 & 0.32 & 0.37 & 0.49 & 0.79 & 0.61 \\
\hline 0.32 & 0.37 & 0.49 & 0.70 & 0.77 & 0.78 & 0.82 \\
\hline 0.37 & 0.49 & 0.70 & 0.77 & 0.81 & 0.78 & 0.82 \\
\hline 0.49 & 0.70 & 0.77 & 0.81 & 0.79 & 0.78 & 0.81 \\
\hline 0.70 & 0.77 & 0.81 & 0.79 & 0.75 & 0.74 & 0.74 \\
\hline 0.77 & 0.81 & 0.79 & 0.75 & 0.67 & 0.71 & 0.70 \\
\hline 0.79 & 0.75 & 0.67 & 0.55 & 0.55 & 0.52 & 0.61 \\
\hline 0.75 & 0.67 & 0.54 & 0.55 & 0.60 & 0.58 & 0.64 \\
\hline 0.67 & 0.55 & 0.55 & 0.60 & 0.64 & 0.64 & 0.72 \\
\hline 0.55 & 0.55 & 0.60 & 0.64 & 0.66 & 0.68 & 0.77 \\
\hline 0.55 & 0.60 & 0.64 & 0.66 & 0.67 & 0.68 & 0.75 \\
\hline 0.65 & 0.66 & 0.67 & 0.79 & 0.83 & 0.78 & 0.81 \\
\hline 0.66 & 0.67 & 0.8 & 0.83 & 0.75 & 0.78 & 0.79 \\
\hline 0.67 & 0.8 & 0.83 & 0.75 & 0.66 & 0.71 & 0.70 \\
\hline 0.8 & 0.83 & 0.75 & 0.66 & 0.60 & 0.63 & 0.65 \\
\hline 0.25 & 0.49 & 0.42 & 0.58 & 0.71 & 0.70 & 0.82 \\
\hline 0.49 & 0.42 & 0.58 & 0.71 & 0.72 & 0.75 & 0.82 \\
\hline 0.72 & 0.64 & 0.53 & 0.63 & 0.66 & 0.67 & 0.73 \\
\hline
\end{tabular}

Table 4 Performance of different neuron models in testing phase for short term load forecasting problem 
International Journal of Artificial Intelligence \& Applications (IJAIA), Vol.2, No.3, July 2011

$\begin{array}{llcc}\text { Model } & \text { RMS Error } & \text { MIN Error } & \text { MAX. Error } \\ \text { Existing } & 0.0692 & 0.0016 & 0.2509 \\ \text { Model 0 } & 0.0679 & 0.0009 & 0.2241 \\ \text { Model 1 } & 0.0671 & 0.0006 & 0.2228 \\ \text { Model 2 } & 0.3918 & 0.0148 & 0.5798 \\ \text { Model 3 } & 0.0694 & 0.0012 & 0.2331 \\ \text { Model 4 } & 0.0692 & 0.0012 & 0.2400 \\ \text { Model 5 } & 0.0681 & 0.0022 & 0.2027 \\ \text { Model 6 } & 0.0695 & 0.0011 & 0.2386\end{array}$

Table 5 Effect of normalisation on training error of short term load

forecasting models (in 32750 eopchs)

$\begin{array}{ccccrrc}\text { Models } & 0.1 \text { to } 1.8 & -0.1 \text { to }-2.7 & -0.9 \text { to }-0.1 & -0.9 \text { to } 0.9 & -1.8 \text { to } 1.8 & -2.7 \text { to } 2.7 \\ 0 & 0.736 & 2.188 & 0.145 & 1.347 & 5.689 & 13.71 \\ 1 & 0.709 & 1.664 & 0.146 & 0.712 & 3.119 & 8.086 \\ 2 & 0.869 & 1.197 & 4.247 & 1.272 & 5.126 & 12.09 \\ 3 & 4.754 & 31.11 & 4.248 & 1.327 & 10.44 & 21.40 \\ 4 & 1.126 & 2.235 & 4.247 & 1.235 & 5.939 & 19.55 \\ 5 & 5.472 & 31.43 & 0.132 & 1.317 & 5.261 & \\ 6 & 4.770 & 31.05 & 4.247 & 1.298 & 6.005 & 20.66\end{array}$

Table 6 RMS error while forecasting the short term demand with different mappings

$\begin{array}{lllll}\text { Model } & \text { X-Y } & \text { DX-Y } & \text { DX-DY } & \text { X-DY } \\ 0 & 0.0679 & 0.6570 & 0.0825 & 0.0852 \\ 1 & 0.0671 & 0.1382 & 0.1036 & 0.0843 \\ 2 & 0.3918 & 0.1458 & 0.1068 & 0.0907 \\ 3 & 0.0694 & 0.1423 & 0.1019 & 0.0964 \\ 4 & 0.0692 & 0.1414 & 0.1011 & 0.0945 \\ 5 & 0.0681 & 0.1402 & 0.1029 & 0.0846 \\ 6 & 0.0695 & 0.1406 & 0.1057 & 0.0941 \\ \text { Existing } & 0.0682 & 0.1953 & 0.1084 & 0.5990\end{array}$

It is well known that the training time and the training error are a function of the range of normalization as well as the input-output mappings. Hence, the effect of normalization on the training error has been studied for the generalized neural network models with X-Y mapping for same number of training epochs (i.e. 32750) and the results are shown in Table 5. The different input output mappings have also been tried to see the effect on training time and error, and the tests results are summarized in Table 6 for musculoskeletal disorders forecasting problem.

\section{CONCLUSION}

This paper brings to the reader different fuzzified neuron models for modeling musculoskeletal disorders forecasting problem. The feed-forward back-propagation neural networks have great 
International Journal of Artificial Intelligence \& Applications (IJAIA), Vol.2, No.3, July 2011

potential in modelling and simulation of these highly nonlinear disorders. We have seen that the training time of neural network models is a function of normalization ranges, different mappings between input-output, the noise in the training data, etc. For musculoskeletal disorder forecasting problem fuzzified neuron model-6 requires minimum training time (i.e. 200 epochs) and it gave minimum error. Model-1 gives least error while forecasting with $\mathrm{X}-\mathrm{Y}$ mapping and the effects of normalization are also minimum on this model. The $\mathrm{X}$ noise-Y mapping gives least error while forecasting using generalized neuron model- 6 . The requirement of the total number of neurons and hidden layers is reduced drastically in the case of the flexible generalized neuron model. The neural networks with the generalized neuron models exhibit much superior properties both in terms of convergence time as well as in terms of prediction error.

\section{REFERENCES}

[1] Alexopoulos EC, Tanagra D, Konstantinou E, Burdorf A. "Musculoskeletal disorders in shipyard industry: prevalence, healthcare use, and absenteeism. BMC Musculoskelet Disord." 2006 Nov 24; 7:88

[2] Alrowayeh HN, Alshatti TA, Aljadi SH, Fares M, Alshamire MM, Alwazan SS. "Prevalence, characteristics, and impacts of work-related musculoskeletal disorders: a survey among physical therapists in the State of Kuwait. BMC Musculoskelet Disord". 2010 Jun 11; 11:116. 10

[3] Briand C, Durand MJ, St-Arnaud L, Corbire M. "Work and mental health: learning from return-to-work rehabilitation programs designed for workers with musculoskeletal disorders.Int J Law Psychiatry.” 2007 Jul-Oct;30(4-5):444-57. Epub 2007 Jul 27.

[4] Burdorf A. "The role of assessment of biomechanical exposure at the workplace in the prevention of musculoskeletal disorders." Scand J Work Environ Health2010 Jan; 36(1):1-2. Epub 2009 Dec 7; pg 29

[5] Carvalho TD, Mozerle A, Ulbrich AZ, Andrade AL, Moraes EE, Mara LS. "Evaluation of the musculoskeletal system of patients in cardiopulmonary and metabolic rehabilitation programs.” Bras Cardio2010 Jul 2

[6] Dash P.K., Dash S., and Rahman S., "A hybrid Neural Network Fuzzy Expert system for Short term load Forecasting”, Elect. Power Res. 25, pp. 149-58, 1992.

[7] Chaturvedi, D.K., Satsangi, P.S. and Kalra, P.K, "Short term Load Forecasting Using Generalized Neural Network Approach", Journal of The Institution of Engineers (India), El, Vol. 74, No. 2, pp. Aug. 1997.

[8] Chaturvedi, D.K., Satsangi, P.S. and Kalra, P.K, "Effect of different mappings and normalization on Artificial neural network models", Ninth National Power Systems Conference at Indian Institute of Technology, Kanpur, India, Dec. 1996.

[9] Descatha A, Chastang JF, Cyr D, Leclerc A, Roquelaure Y, Evanoff B. "Do workers with self-reported symptoms have an elevated risk of developing upper extremity musculoskeletal disorders three years later? Occup Environ” Med2008 Mar;65(3):205-7. Epub 2007 Aug 31.

[10] Dillon T.S., Sestito S. and Leng S., "Short-term load Forecasting Using Adaptive Neural Network", Electric Power Energy System 13 (4), pp. 186-92, 1991.

[11] Doderlein L Importance of the muscular system in the development of neuromuscular deformities: new diagnostic and therapeutic aspects Orthopade2010 Jan; 39(1):7-14.pg 23 
International Journal of Artificial Intelligence \& Applications (IJAIA), Vol.2, No.3, July 2011

[12] Drobnic F, Turmo A. "Hyperbaric oxygen treatment of musculoskeletal disorders on the sports medicine. State of the art Med Clin (Barc)". 2010 Mar 13; 134(7): 312-5. Epub 2009 Nov 22.

[13] Essaddam $\mathrm{H}$ "The use of joint cartilage in the anatomical classification of bones and its use in the teaching and therapeutic management of skeleton disorders" Tunis Med2009 Dec; 87(12):891-3pg 19

[14] Fassa AG, Facchini LA, Dall'Agnol MM, Christiani DC. "Child labor and musculoskeletal disorders: the Pelotas (Brazil) epidemiological survey." Public Health Rep. 2005 Nov-Dec; 120(6):665-73."

[15] Fernandes Rde C, Assun $\pi$ o AA, Silvany Neto AM, "Carvalho FMMusculoskeletal disorders among workers in plastic manufacturing plants.” Rev Bras Epidemiol2010 Mar;13(1):11-20.

[16] Flanagan AM, Delaney D, O'Donnell P. "Benefits of molecular pathology in the diagnosis of musculoskeletal disease: Part II of a two-part review: bone tumors and metabolic disorders Skeletal Radiol”.2010 Mar;39(3):213-24. Epub 2009 Aug 11.pg 38

[17] Huisstede BM, van Middelkoop M, Randsdorp MS, Glerum S, Koes BW.Effectiveness of interventions of specific complaints of the arm, neck, and/or shoulder: 3 musculoskeletal disorders of the hand. An updateArch Phys Med Rehabi2010 Feb; 91(2):298-314.; pg 21

[18] Jang Y, Chi CF, Tsauo JY, Wang JD. "Prevalence and risk factors of work-related musculoskeletal disorders in massage practitioners.” J Occup Rehabil. 2006 Sep; 16(3):425-38

[19] Jordan KP, Kadam UT, Hayward R, Porcheret M, Young C, Croft P. "Annual consultation prevalence of regional musculoskeletal problems in primary care: an observational study" BMC Musculoskelet Disord2010 Jul 2;11:144

[20] Jull G, Amiri M, Bullock-Saxton J, Darnell R, Lander C. "Cervical musculoskeletal impairment in frequent intermittent headache. Part 1: Subjects with single headaches" Cephalalgia2007 Jul; 27(7):793-802.

[21] Kim K.H., Park D.V., Park J.K., "A hybrid model of Artificial Neural Network and Fuzzy Expert Systems for Short term load Forecasting”, Proc. IV Symp : Expert System Application to Power System, Melbourne Australia, pp. 159-65, 1993.

[22] Lee, K.Y., Cha Y.T. and Park J.H.,"Artificial Neural Network methodology for short term load forecasting", NSF Workshop on Artificial Neural Network Methodology in Power systems Engineering, Clemson University, SC, Apr. 9-10, 1990.

[23] Lippmann, R. "An Introduction to Computing with Neural Networks", IEEE ASSP Magazine, pp. 4-22, April 1987.

[24] Lipmann, R.P., "Pattern Classification Using Neural Networks", IEEE Communications Magazine, Nov., 1989.

[25] Mizumoto, M., "Pictorial Representations of Fuzzy Connectives, Part II: Cases of Compensatory Operators and Self - Dual Operators", Fuzzy Sets and Systems, 32, NorthHolland, pp. 45-79, 1989.

[26] Rumelhart D.E., Hinton G.E. and Willams R.J., "Learning Internal representation by error Propagation", Parallel Distributed Processing, Vol. 1, pp. 318-62, Cambridge, MA:MIT Press, 1988. 
International Journal of Artificial Intelligence \& Applications (IJAIA), Vol.2, No.3, July 2011

[27] Singh, A., Emmamel P. and Kalra P.K., "Point of view for development of knowledge based systems for Load Forecasting", Proc. 1st. Symp. Expert Systems Application to Power System, Stockholm, Sweden and Helsinki, Finland, pp. 7/1-7/4., 1988.

[28] Srinivasan D., Liew A.C., and Cheng, C.S., "A neural Network Short term load Forecasting", Electric Power System Research 28, pp. 227-34, 1994.

[29] Wasserman, P.D., Neural Computing: Theory and Practice, Van Nostrand Reinhold, New York, 1966.

[30] Webb, A.R., "Functional Approximation by feed Forward Network: A least Square Approach to Generalization”, IEEE Trans. on Neural Networks, Vol. 5, May, pp. 363-71, 1994.

[31] Widrow B. and Lehr M.A. "30 Years of Adaptive Neural Networks: Perceptron, Madaline and Backpropagation”, Proceedings of IEEE, 78 (9), pp. 1415-42, 1990.

\section{AUTHORS}

Aman Sachdeva is an Assistant Professor in Department of Mechanical Engineering at Anand Engineering College, Agra. His professional qualification includes an M.Tech in Engineering Systems from Dayalbagh Educational Institute, Agra. His area of research has been Ergonomics and development of Artificial Intelligent Tools.

Prof. B.D.Gupta is a Director at Anand Engineering College, Agra. His professional qualification includes a Doctorate in Mechanical Design from Indian Institute of Technology, Kanpur and an M.Tech in Mechanical Engineering also from Indian Institute of Technology, Kanpur. His area of research is Mechanical Design, Alternative Source of Energy and Operations Research.

Prof Sneh Anand is a Professor in Centre for Bio-Medical Engineering at Indian Institute of Technology, Delhi. Her professional qualification includes a Doctorate from Indian Institute of Technology, Delhi and her area of research is Biomedical Instrumentation and rehabilitation engineering. 